\title{
Anisotropy of Electrical Resistivity in High-Purity Aluminium Single Crystals
}

\author{
Y. Ueda, E. Hashimoto, H. Tamura and T. Kino* \\ Laboratory of Crystal Physics, Faculty of Science, Hiroshima University, Higashi-Hiroshima 739, \\ Japan \\ * Hiroshima-Denki Institute of Technology, Hiroshima 739-03, Japan
}

\begin{abstract}
The current direction dependence of electrical resistivity was measured at temperatures between 4.2 and $80 \mathrm{~K}$ in single crystals of high-purity aluminium (RRR $\sim 50000$ ) with a common $(110\}$ surface. The main results found in this experiment are as follows. (1) The residual resistivity in the bulk value, $\rho_{b}$, shows high dependence on current direction, basically increasing, in order, in the $\langle 110\rangle,\langle 111\rangle$ and $\langle 001\rangle$ directions. This anisotropy increases in purer specimens. Moreover, current direction dependence shows a salient structure that corresponds to the structure of the Fermi surface of aluminium. (2) In the temperature region below $25 \mathrm{~K}$, the temperature dependent part of the resistivity also shows the same features as the anisotropy of $\rho_{b}$. At higher temperatures than $25 \mathrm{~K}$, this anisotropy disappears as the phonon resistivity increases.
\end{abstract}

\section{INTRODUCTION}

Highly pure aluminium has been developed in our laboratory by the zone refining method [1,2]. Recently, its residual resistance ratioRRR $(=\mathrm{R}(300 \mathrm{~K}) / \mathrm{R}(4.2 \mathrm{~K})$ ) has reached about 100000 . In such a pure metal, the mean free path of conduction electrons becomes 2-3 $\mathrm{mm}$ at low temperatures. At the same time, the relaxation time and the phase coherence length are also enlarged. Then, unknown properties would emerge especially in electronic transport phenomena such as the electrical resistivity, its temperature dependence, the magnetoresistance and so on.

Recently, we found that the bulk residual resistivity $\rho_{b}$ at $4.2 \mathrm{~K}$ shows anisotropy with respect to the current direction or electric field direction in aluminium single crystals ( RRR $\sim 50000$ ) with a common $\{110\}$ surface [3]. The bulk resistivity $\rho_{b}$ in-creased, in order, in the $\langle 110\rangle,\langle 111\rangle$ and $<001>$ current directions. This is a remarkable result since the conductivity tensor is believed to become a scalar in cubic crystals [4 ]. In order to reveal the mechanism of this anisotropy, we carried out more detailed experiments on the dependence of the resistivity on specimen thickness, current direction and temperature.

\section{EXPERIMENTAL PROCEDURE}

Single-crystal specimens $0.2-1 \mathrm{~mm}$ in thickness, $3 \mathrm{~mm}$ in width and 7-30 $\mathrm{mm}$ in length were cut with a spark erosion machine. They were prepared from the close part of the aluminium single-crystal rod (RRR 50 000) produced by zone refining. The main surface of all the specimens was cut parallel with $\{110\}$ in order to keep the surface scattering of conduction electrons identical. The surfacenormal and the axis orientation of the specimens were determined by the transmission Laue method and accurate within $\pm 1^{\circ}$. These specimens were chemically etched with aqua regia before and after the spot-welding of four zone-refined aluminium wires $0.3 \mathrm{~mm}$ in diameter as the electrodes for the DC four-probe measurement. They were annealed at $300^{\circ} \mathrm{C}$ in air for $3 \mathrm{~h}$ and cooled down to room temperature in an electric furnace. These specimens were carefully placed on a holder without strain. A 
superconducting chopper amplifier with the optimum resolution of $2 \mathrm{pV}$ [5] was used for the residual resistance measurement at $4.2 \mathrm{~K}$ and a direct current comparator potentiometer (Guildline, Model 9930) with a resolution of $1 \mathrm{nV}$ for the measurement of the temperature dependence of resistance.

The residual resistivity at $4.2 \mathrm{~K}, \rho(4.2 \mathrm{~K})$, was determined from the relation $\rho(4.2 \mathrm{~K})=[\rho(300$ $\mathrm{K}) / \mathrm{R}(300 \mathrm{~K})] \mathrm{R}(4.2 \mathrm{~K})$, where $\mathrm{R}(300 \mathrm{~K})$ and $\mathrm{R}(4.2 \mathrm{~K})$ are resistances of the specimens measured at $300 \mathrm{~K}$ and $4.2 \mathrm{~K}$, respectively and $\rho(300 \mathrm{~K})$ is the resistivity at $300 \mathrm{~K}$. A value of $27.33 \mathrm{n} \Omega \mathrm{m}$ was used for $\rho(300 \mathrm{~K})$. The specimen thickness $d$ was determined from the relation $d=[\rho(300 \mathrm{~K}) / \mathrm{R}(300$ $K)] \times\left(l_{p} / w\right)$, where $l_{p}$ is the distance between potential electrodes and $w$ the width of the specimens. A travelling microscope with the resolution of $\pm 0.01 \mathrm{~mm}$ was used for the measurement of $\mathrm{l}_{\mathrm{p}}$ and $w$.

In the measurement of temperature dependence, one pair of specimens were mounted on a copper disk in order to obtain a resistivity difference between two specimens. The copper disk was attached on a helium pot in a cryostat. The cryostat was spontaneously warmed from 4.2 to $80 \mathrm{~K}$ during about $30 \mathrm{~h}$ to keep aniform temperature distribution in the copper disk. Temperatures were monitored with a thermocouple of Au- 0.07 at.\% Fe vs. chromel placed between the pair of specimens to an accuracy of $\pm 0.07 \mathrm{~K}$. A small potential difference $\Delta \mathrm{V}$ between these specimens was measured with the $D C$ comparator potentiometer. The resistance ratio was determined from the relation $R_{2} / R_{1}=\left(I_{1} / I_{2}\right)[1+$ $\left.\Delta V /\left(I_{1} R_{1}\right)\right]$, where $R_{1}$ and $R_{2}$ are the resistance of a reference specimen and an unknown specimen, respectively, and $I_{1}$ and $I_{2}$ are currents through $R_{1}$ and $R_{2}$, respectively. The specimen with the $<111>$ axis was used as the reference specimen.

\section{RESULTS AND DISCUSSION \\ 3.1 Thickness dependence of $p(4.2 \mathrm{~K})$}

The specimen axis along which a current flew was directed in one of three principal orientations $<110\rangle,<111>$ and $<100>$. Figure 1 shows the experimental values of $\rho(4.2 \mathrm{~K})$ as a function of $\mathrm{P} / 2 \mathrm{~A}$, where $P$ is the perimeter of a cross section and $A$ the sectional area of a specimen. The dependence of $\rho(4.2 \mathrm{~K})$ on the orientation of the specimen axis is distinct for $\mathrm{P} / 2 \mathrm{~A}<3 \times 10^{3} \mathrm{~m}^{-1}$ : the resistivity increases in the order of the $\langle 110\rangle,\langle 111\rangle$ and $\langle 100\rangle$ directions. This anisotropy of $\rho(4.2 \mathrm{~K})$ decreases in thin specimens in which the scattering rate of conduction electrons at the specimen surface is large. As the thickness of specimens decreases, the $\rho(4.2 \mathrm{~K})$ values of the $\langle 111\rangle$ and $<100\rangle$ specimens rapidly approach the $\rho(4.2 \mathrm{~K})$ value of the $<110>$ specimens for $\mathrm{P} / 2 \mathrm{~A}>3 \times 10^{3} \mathrm{~m}^{-1}$ and $>$ $4 \times 10^{3} \mathrm{~m}^{-1}$, respectively, reducing their increment with $\mathrm{P} / 2 \mathrm{~A}$. These results suggest that the anisotropic distribution of electrons in an applied electric field is homogenized by the increased surface scattering.

If the anisotropy of $\rho(4.2 \mathrm{~K})$ with the axis orientations arises from a break of the cubic symmetry due to impurities aligned in a certain direction, or due to defects and microstructure produced in the surface layers during spark-cutting, the anisotropy will remain constant or increase in thin specimens. As the thickness of specimens decreases, the volume fraction of the defects and microstructure becomes large, resulting in increased resistivity. Therefore, the result in Figure 1 that the anisotropy decreases in thin specimens shows that the impurities, the defects and the microstructure are not the cause of this anisotropy.

Solid curves in Figure 1 are drawn through the data points for each axis orientation according to the Fuchs-Sondheimer theory [6]. As the specimens were etched with aqua regia and annealed in air at $300^{\circ} \mathrm{C}$, the surface of the specimens is rather rough. Therefore, the surface scattering of conduction electrons was assumed to be diffuse, i.e., the specularity parameter $p=0[7]$. A value of $0.82 \mathrm{f} \Omega \mathrm{m} 2$ was used for the product of bulk resistivity and bulk mean free path [7,8]. Each of these curves gives a good fit to the data obtained, except those for the thin $\langle 100\rangle$ and $<111>$ specimens and for the thickest $<111>$ and $<110>$ specimens.

In thick specimens, the surface scattering on the side surface becomes large. The side surface of the $<100\rangle$ specimen has the same $\{110\}$ crystallographic plane as the main surface. But the $\langle 111\rangle$ and $<110>$ specimens have the $\{112\}$ and $\{100\}$ side surfaces, respectively. These side surfaces may give the different size effect from that due to the $\{110\}$ main surface. This effect must necessarily be pronounced in thick specimens. For example, the value of $\rho(4.2 \mathrm{~K})$ for the thickest $\langle 110\rangle$ specimen suggests that the size effect due to the $\{100\}$ surface is significantly larger than that due to the $\{110\}$ 
surface. This is consistent with previous experiments on the anisotropic size effect in thin aluminium films [9]. If such a contribution from the side surface is negligible to the total resistivity, the FuchsSondheimer theory with a suitable value of $\rho_{b} l_{b}$ as described above will be plausible to estimate the bulk residual resistivity, $\rho_{\mathrm{b}}$, at $4.2 \mathrm{~K}$ for the specimens studied.

The salient feature of the results shown in Figure 1 is the anisotropy of the bulk residual resistivity $\rho_{b}$. The resistivity increases in the order of the $\langle 110\rangle,\langle 111\rangle$ and $\langle 100\rangle$ directions: the values along $<100>$ and $<111>$ are, respectively, $50 \%$ and $20 \%$ higher than those along $<110>$. This anisotropy increased in purer specimens: in specimens with RRR -10000 , the anisotropy was less than 6\%; in specimens with $R R R \sim 100000$, the values along $<100>$ and $<111>$ were, respectively, $70 \%$ and $30 \%$ larger than those along $<110$. This result is noteworthy for the transport phenomena of electrons because aluminium is a normal metal and has the cubic symmetry. On this condition, it was generally believed that the resistivity is isotropic [4].

The linearity of an electric field $\mathrm{E}$ against a current density $\mathrm{i}$ was confirmed within the experimental error for all the specimens at $4.2 \mathrm{~K}$ for a current from $0.08 \mathrm{~A}$ up to $1.2 \mathrm{~A}$. This linear behaviour means that the possibilities of the influence of Joule heat and magnetoresistance that increases as $\mathrm{i}^{2}$ are discarded. Therefore, we conclude that the anisotropy of $\rho_{b}$ obtained in this work is the intrinsic bulk property under an influence of the electric field.

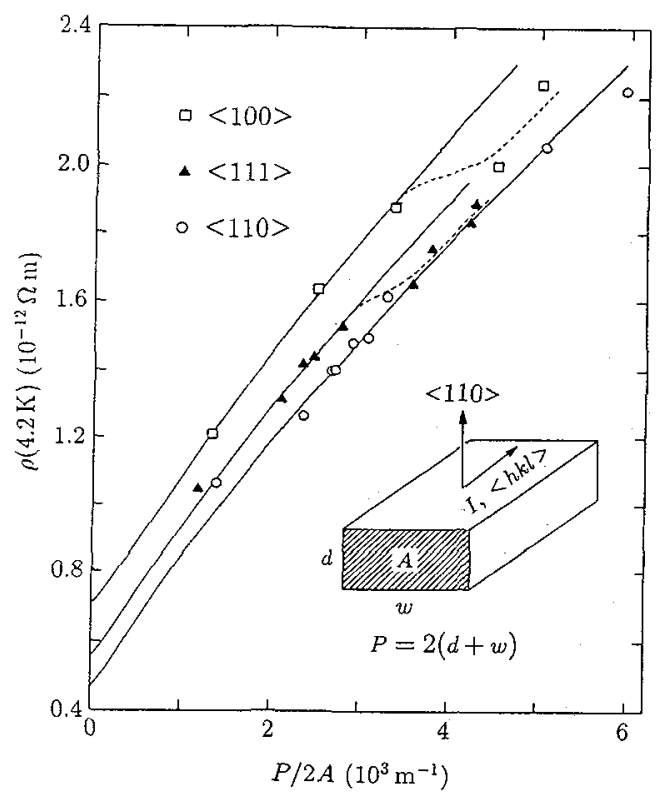

Figure 1: Experimental values of $\rho(4.2 \mathrm{~K})$ as a function of $P / 2 A$. Here, $P$ is the perimeter of a cross section and $A$ the sectional area of a specimen. The notation $\langle h k l\rangle$ represents the orientation of the specimen axis. The orientation of the main surface is $\{110\}$ for all the specimens. Solid curves are obtained according to the Fuchs-Sondheimer theory assuming that $p=0$ and $\rho_{\mathrm{b}} \ell_{\mathrm{b}}=$ $0.82 \mathrm{f} \Omega \mathrm{m}^{2}$. Dashed curves are drawn to guide the eye.

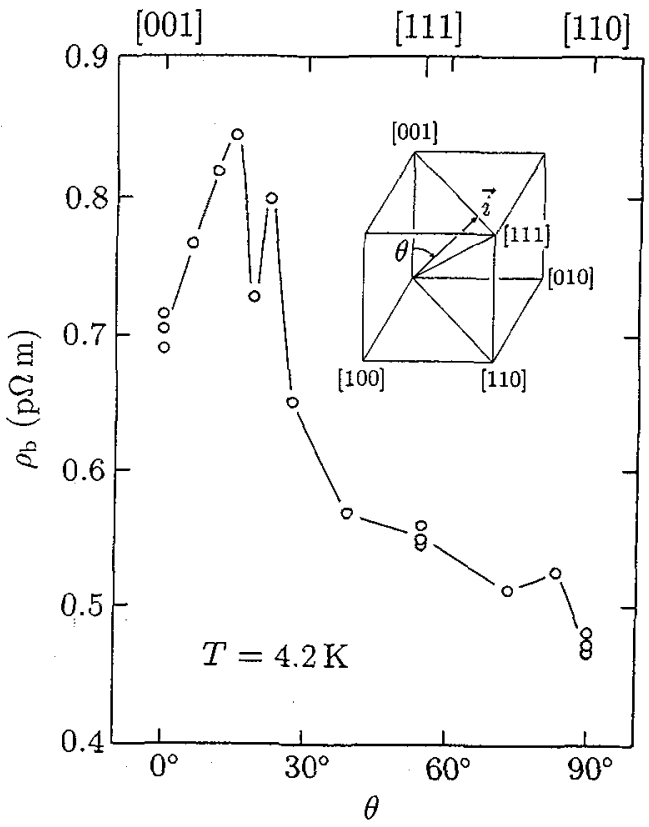

Figure 2: The bulk resistivity $\rho_{\mathrm{b}}$ at $4.2 \mathrm{~K}$ as a function of the angle $\theta$ between [001] and the specimen axis along which an electric current flows. The solid line is drawn to guide the eye. The inset explains the angle $\theta$. 


\subsection{Current direction dependence of $\rho_{b}$}

Figure 2 shows the bulk resistivity $\rho_{\mathrm{b}}$ as a function of the angle $\theta$. The thickness of the specimens was confined to values between 0.3 and $0.6 \mathrm{~mm}$ in the light of the results in section 3.1. However, a thicker specimen with the [001] axis direction was included since the side surface has the same orientation $\{110\}$ as the main surface.

The $\theta$-dependence of $\rho_{b}$ is not monotonous, but shows a large structure around $\theta=20^{\circ}$ and a small one around $\theta=85^{\circ}$. As $\theta$ increases, $\rho_{b}$ increases still more from the value at $\theta=0^{\circ}$, where the specimen axis is parallel with [001]. The value of $\rho_{b}$ at $\theta=15^{\circ}$ is $80 \%$ larger than that at $\theta=90^{\circ}$ corresponding to the [110] specimens. With further increasing $\theta$, the bulk resistivity $\rho_{b}$ shows a dip around $\theta=20^{\circ}$, and then it decreases monotonously until $\theta$ reaches $90^{\circ}$ except a small increase around $\theta=85^{\circ}$.

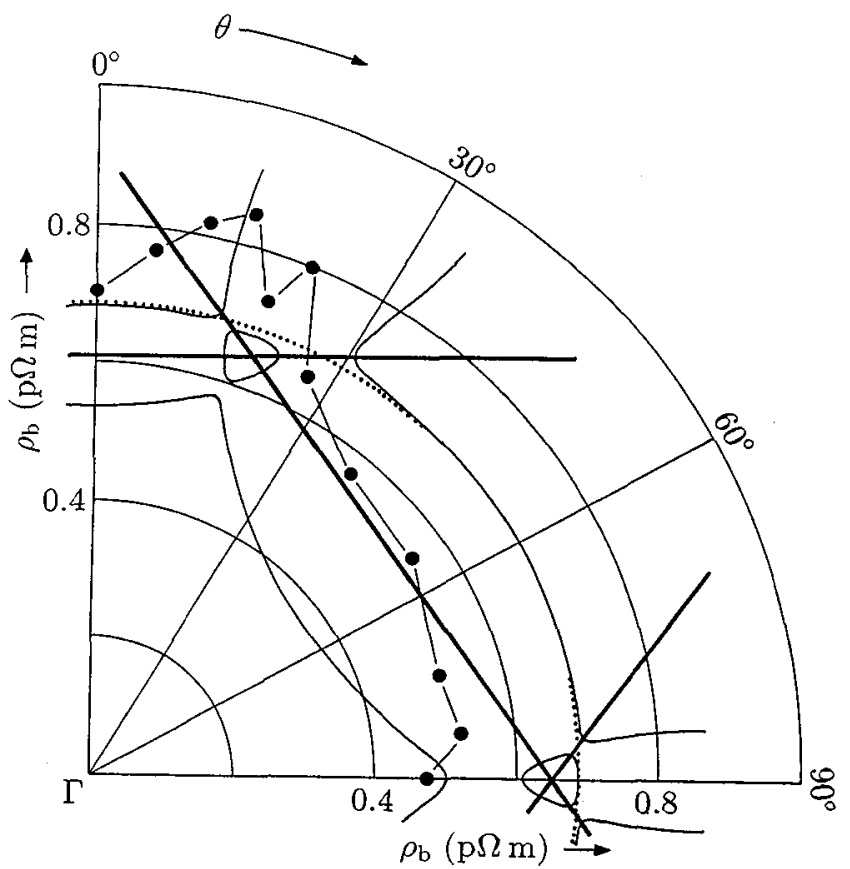

Figure 3: Polar plot of $\rho_{\mathrm{b}}$ stuck on the central (1ī0) cross section of the Fermi surface of aluminium. For clearness, the average value is plotted for the [001], [111] and [110] specimens. The thick lines show the Brillonin zone boundaries and the dotted curve represents the Fermi sphere.

The behaviour of the $\theta$-dependence of $\rho_{b}$ suggests some correlation with the detailed structure of the Fermi surface of aluminium. In order to see this correlation, we plot in Figure 3 the $\theta$-dependence of $\rho_{b}$

on the polar coordinate stuck on the $(1 \overline{1} 0)$ cross section of the Fermi surface that includes the point. We calculated this cross section according to the 4-OPW method using the pseudopotentials obtained by Joss and Monnier [10]. A strong correlation between the anisotropy of the bulk resistivity $\rho_{b}$ and the structure of the Fermi surface is clearly found. The value of $\rho_{b}$ increases at $\theta$ nearly in the direction that the Fermi sphere crosses the Brillouin zone boundary. Around the [111] direction $\left(\theta=54.7^{\circ}\right)$ where a conduction electron is almost free-electron-like, the variation of $\rho_{b}$ is weak. It is further noted that $\rho_{b}$ is not enhanced in the direction towards the corners of Fermi surface in the second zone where the Fermi velocity of an electron is smaller than that in other regions that lie away from the zone boundaries [11]. The experimental value of $\rho_{\mathrm{b}}$ is smallest in the [110] direction. 
The results as shown in Figure 3 suggest that the scattering probability of conduction electrons greatly increases when the electric field is applied around the direction that the Fermi sphere crosses the Brillouin zone boundary, especially in the direction to the $U$ point on the zone boundary. The Fermi surface of aluminium is closest to the zone boundary perpendicular to [001] and involves the $\mathrm{X}$, $\mathrm{W}$ and $U$ points. Near the $W$ point, the Fermi surface in the second zone contacts with that in the third zone in the 4-OPW calculation. When the electric field is applied, the electron distribution is shifted along the electric field, resulting in deviation from the equilibrium state. This deviation will depend on the direction of the electric field due to the anisotropic structure of the Fermi surface of aluminium. The anisotropic deviation of the electron distribution may enhance the scattering probability and induce the strong anisotropy of the resistivity.

\subsection{Temperature dependence of resistivity}

Specimens with the principal axes $\langle 110\rangle,\langle 111\rangle$ and $\langle 100\rangle$ were also prepared from the singlecrystal rod. The ratios $\rho_{100}^{\text {ph }}(\mathrm{T}) / \rho_{111}^{\mathrm{h}}(\mathrm{T})$ and $\rho_{110}^{\text {th }}(\mathrm{T}) / \rho_{111}^{\mathrm{hh}}(\mathrm{T})$ are shown in Figure 4 , where $\rho_{\mathrm{hkl}}^{\text {th }}(\mathrm{T})$ means the temperature-dependent part of the resistivity $\rho^{\text {ph }}(\mathrm{T})$ in a specimen with the $\langle\mathrm{hkl}\rangle$ axis. In order to obtain $\rho^{\mathrm{ph}}(T)$, the residual part $\rho(0)$ was subtracted from the total resistivity $\rho(T)\left(=\rho(0)+\rho^{\mathrm{ph}}(\mathrm{T})\right)$. The value of $\rho(0)$ was determined by the least mean squares fitting of a polynomial, $A_{0}+A_{2} T^{2}+A_{3} T^{3}$ $+A_{5} T^{5}$, to the data of $\rho(T)$ obtained below $15 \mathrm{~K}$. The deviation of the ratios from 1.0 represents the anisotropy of the temperature dependence of resistivity.

A remarkable anisotropy is observed in $p^{\text {ph }}(\mathrm{T})$ at low temperatures. At temperatures below about 25 $K, \rho_{100}^{\text {ph }}(T)$ is larger than $\rho_{111}^{\text {ph }}(T)$, but $\rho_{110}^{\text {ph }}(T)$ is oppositely smaller than $\rho_{111}^{\text {ph }}(T): \rho_{100}^{\text {ph }}(T)>\rho_{111}^{\text {ph }}(T)>$ $\rho_{110}^{\mathrm{ph}}(\mathrm{T})$. In this temperature region, $\rho^{\mathrm{ph}}(\mathrm{T})$ has the larger value in the order of symmetry of the specimen axis. These features are the same as those of $\rho_{\mathrm{b}}$. The ratio $\rho_{110}^{\mathrm{ph}}(\mathrm{T}) / \rho_{111}^{\text {h }}(\mathrm{T})$ approaches rapidly to 1.0 with increasing temperature. On the other hand, the ratio $\rho_{100}^{\text {th }}(T) / \rho_{111}^{\text {th }}(T)$ approximates to 1.0 at about $25 \mathrm{~K}$ and takes the minimum value between 30 and $35 \mathrm{~K}$. Then, it gradually approaches 1.0 at higher temperatures. The relative difference of $\rho^{\text {ph }}(T)$ between specimens with the different axis orientation is large at temperatures below $20 \mathrm{~K}$, and this large anisotropy seems to persist even in the lowest temperature region although the data are scattered.

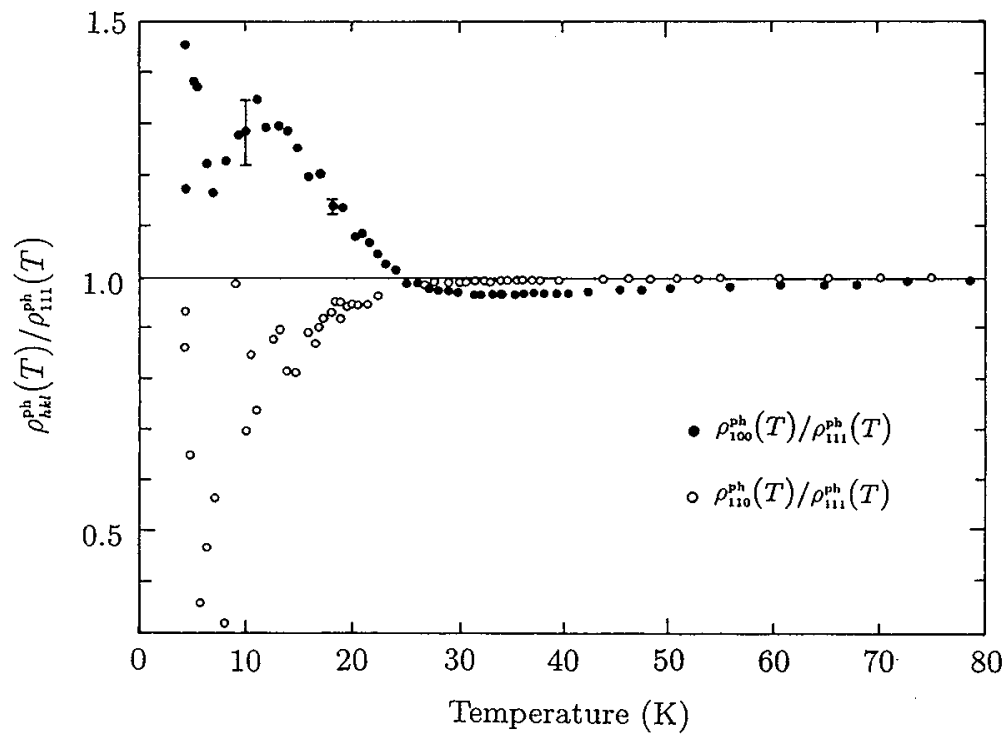

Figure 4: Ratios of the temperature dependent part of electrical resistivity $\rho_{h k l}^{\mathrm{ph}}(T)$ to $\rho_{111}^{\mathrm{ph}}(T)$ as a function of temperature for the aluminium single-crystal specimens with the $\langle h k l\rangle$ axis. 
The behaviour of the anisotropy of $\rho^{\mathrm{ph}}(\mathrm{T})$ at $\mathrm{T}<25 \mathrm{~K}$ shows a good correspondence with that of $\rho(4.2 \mathrm{~K})$ as a function of $\mathrm{P} / 2 \mathrm{~A}$ in Figure 1. The anisotropy of $\rho^{\mathrm{ph}}(\mathrm{T})$ in the low temperature region, where the rate of the electron-phonon scattering is small, is the same as that of $\rho(4.2 \mathrm{~K})$ in the region $\mathrm{P} / 2 \mathrm{~A}<3 \times 10^{3} \mathrm{~m}^{-1}$. In the higher temperature region, where the scattering rate increases, the anisotropy of $\rho^{\text {ph }}(\mathrm{T})$ decreases similarly to that of $\rho(4.2 \mathrm{~K})$ in the region $\mathrm{P} / 2 \mathrm{~A}>4 \times 10^{3} \mathrm{~m}^{-1}$.

In high-purity aluminium for the present experiment, the electron-impurity scattering is largely reduced. Eventually, the anisotropic electron-phonon scattering at low temperatures and the electronelectron scattering relatively increase their influence on the electrical resistivity. In fact, $\rho^{\text {ph }}(T)$ in the present specimens deviates from the Bloch-Grüneisen expression at temperatures below $20 \mathrm{~K}$ where the anisotropy of $\rho^{\mathrm{ph}}(T)$ is remarkable. In aluminium, the second-zone Fermi surface is nearest to the Brillouin zone boundary that is perpendicular to the high-symmetric direction $<100\rangle$.

When an electric field is applied in the $\langle 100\rangle$ direction, the electron distribution deviates from equilibrium in this direction towards the zone boundary. A possible suggestion to explain the anisotropy of $\rho^{\text {ph }}(T)$ is that the probability of the umklapp process is enhanced due to this deviation of electron distribution by the electric field in $\langle 100\rangle$. When the temperature is raised over $20 \mathrm{~K}$, the scattering rate of electrons due to the normal process increases and the electron distribution will be homogenized. Then, the anisotropy of $\rho^{\mathrm{ph}}(\mathrm{T})$ will decreases. The change in the process of electronphonon scattering will be a cause of the detailed behaviour of $\rho_{100}^{\mathrm{ph}}(T) / \rho_{11}^{\mathrm{ph}}(\mathrm{T})$ in Figure 4 that shows the minimum between 30 and $35 \mathrm{~K}$. The mechanism of the anisotropy that works in $\rho^{\text {ph }}(\mathrm{T})$ may relate to that of the residual resistivity. Further investigations, experimental and theoretical, are needed to elucidate the mechanism of the anisotropy of electrical resistivity.

\section{References}

[1] Kino T., Kamigaki N., Yamasaki H., Kawai J., Deguchi Y. and Nakamichi I., Trans. Jpn. Inst. Met. 17 (1976) 645.

[2] Hashimoto E., Ueda $Y$. and Kino T., This Conference.

[3] Hashimoto E., Ueda Y., Tamura H. and Kino T., J. Phys. Soc. Japan 62 (1993) 4178.

[4] Rossiter P. L., The electrical resistivity of metals and alloys (Cambridge University Press, Cambridge, 1986) chap. 1.

[5] Ueda Y., Hosoda H. and Kino T., J. Phys. Soc. Japan 57 (1988) 3896.

[6] Sondheimer E. H., Adv. Phys. 1 (1952) 1.

[7] Sambles J. R., Elsom K. C. and Sharp-Dent G., J. Phys. F: Met. Phys. 11 (1981) 1075.

[8] Ueda Y., Hosoda H. and Kino T., J. Phys. Soc. Japan 54 (1985) 3858.

[9] Sato H. and Yonemitsu K., Phys. Status Solidi b 73 (1976) 723.

[10] Joss W. and Monnier R., J. Phys. F: Met. Phys. 10 (1980) 9.

[11] Böning K., Pfändner K., Rosner P. and Schlüter M., J. Phys. F: Met. Phys. 5 (1975) 1176. 